\title{
Defining the minimum clinically important difference for grade I degenerative lumbar spondylolisthesis: insights from the Quality Outcomes Database
}

\author{
Anthony L. Asher, MD, ${ }^{1}$ Panagiotis Kerezoudis, MD, ${ }^{2}$ Praveen V. Mummaneni, MD, ${ }^{3}$ \\ Erica F. Bisson, MD, MPH, ${ }^{4}$ Steven D. Glassman, MD, ${ }^{5}$ Kevin T. Foley, MD, ${ }^{6}$ \\ Jonathan R. Slotkin, MD, ${ }^{7}$ Eric A. Potts, MD, ${ }^{8}$ Mark E. Shaffrey, MD, ${ }^{9}$ Christopher I. Shaffrey, MD, ${ }^{9}$ \\ Domagoj Coric, MD, ${ }^{1}$ John J. Knightly, MD, ${ }^{10}$ Paul Park, MD, ${ }^{11}$ Kai-Ming Fu, MD, PhD, ${ }^{12}$ \\ Clinton J. Devin, MD, ${ }^{13}$ Kristin R. Archer, PhD, DPT, ${ }^{13,14}$ Silky Chotai, MD, ${ }^{13}$ Andrew K. Chan, MD, ${ }^{3}$ \\ Michael S. Virk, MD, PhD, ${ }^{12}$ and Mohamad Bydon, MD²
}

\begin{abstract}
'Department of Neurosurgery, Carolina Neurosurgery and Spine Associates and Neuroscience Institute, Carolinas HealthCare System, Charlotte, North Carolina; ${ }^{2}$ Department of Neurologic Surgery, Mayo Clinic, Rochester, Minnesota; ${ }^{3}$ Department of Neurologic Surgery, University of California, San Francisco, California; ${ }^{2}$ Department of Neurologic Surgery, University of Utah, Salt Lake City, Utah; ${ }^{5}$ Norton Leatherman Spine Center, Louisville, Kentucky; ${ }^{6}$ Department of Neurologic Surgery, University of Tennessee, Memphis, Tennessee; ${ }^{13}$ Department of Orthopedic Surgery, Vanderbilt University; ${ }^{14}$ Department of Physical Medicine and Rehabilitation, Vanderbilt University Medical Center, Nashville, Tennessee; ${ }^{7}$ Geisinger Health, Danville, Pennsylvania; ${ }^{8}$ Department of Neurologic Surgery, Indiana University; Goodman Campbell Brain and Spine, Indianapolis, Indiana; ${ }^{9}$ Department of Neurologic Surgery, University of Virginia Health System, Charlottesville, Virginia; ${ }^{10}$ Atlantic Neurosurgical Specialists, Morristown, New Jersey; ${ }^{11}$ Department of Neurologic Surgery, University of Michigan, Ann Arbor, Michigan; and ${ }^{12}$ Department of Neurologic Surgery, Weill Cornell Medical College, New York, New York
\end{abstract}

OBJECTIVE Patient-reported outcomes (PROs) play a pivotal role in defining the value of surgical interventions for spinal disease. The concept of minimum clinically important difference (MCID) is considered the new standard for determining the effectiveness of a given treatment and describing patient satisfaction in response to that treatment. The purpose of this study was to determine the MCID associated with surgical treatment for degenerative lumbar spondylolisthesis.

METHODS The authors queried the Quality Outcomes Database registry from July 2014 through December 2015 for patients who underwent posterior lumbar surgery for grade I degenerative spondylolisthesis. Recorded PROs included scores on the Oswestry Disability Index (ODI), EQ-5D, and numeric rating scale (NRS) for leg pain (NRS-LP) and back pain (NRS-BP). Anchor-based (using the North American Spine Society satisfaction scale) and distribution-based (half a standard deviation, small Cohen's effect size, standard error of measurement, and minimum detectable change [MDC]) methods were used to calculate the MCID for each PRO.

RESULTS A total of 441 patients ( 80 who underwent laminectomies alone and 361 who underwent fusion procedures) from 11 participating sites were included in the analysis. The changes in functional outcome scores between baseline and the 1-year postoperative evaluation were as follows: $23.5 \pm 17.4$ points for ODI, $0.24 \pm 0.23$ for EQ-5D, $4.1 \pm 3.5$ for NRS-LP, and $3.7 \pm 3.2$ for NRS-BP. The different calculation methods generated a range of MCID values for each PRO: 3.3-26.5 points for ODI, 0.04-0.3 points for EQ-5D, 0.6-4.5 points for NRS-LP, and 0.5-4.2 points for NRS-BP. The MDC approach appeared to be the most appropriate for calculating MCID because it provided a threshold greater than the measurement error and was closest to the average change difference between the satisfied and not-satisfied patients. On subgroup analysis, the MCID thresholds for laminectomy-alone patients were comparable to those for the patients who underwent arthrodesis as well as for the entire cohort.

CONCLUSIONS The MCID for PROs was highly variable depending on the calculation technique. The MDC seems to be a statistically and clinically sound method for defining the appropriate MCID value for patients with grade I degenera-

ABBREVIATIONS AUC = area under the curve; $\mathrm{HTI}=$ health transition item; $\mathrm{MCID}=$ minimum clinically important difference; $\mathrm{MDC}=$ minimum detectable change; $\mathrm{NASS}$ = North American Spine Society; NRS-BP = numeric rating scale for back pain; NRS-LP = NRS for leg pain; ODI = Oswestry Disability Index; PRO = patient-reported outcome; $\mathrm{QOD}=$ Quality Outcomes Database; ROC = receiver operating characteristic; $\mathrm{SD}=$ standard deviation; SEM = standard error of measurement; TLIF = transforaminal interbody fusion; VAS = visual analog scale.

SUBMITTED August 31, 2017. ACCEPTED October 19, 2017.

INCLUDE WHEN CITING DOI: 10.3171/2017.10.FOCUS17554. 
tive lumbar spondylolisthesis. Based on this method, the MCID values are 14.3 points for ODI, 0.2 points for EQ-5D, 1.7 points for NRS-LP, and 1.6 points for NRS-BP.

https://thejns.org/doi/abs/10.3171/2017.10.FOCUS17554

KEY WORDS lumbar; spondylolisthesis; Quality Outcomes Database; MCID; minimum clinically important difference; quality of life; patient-reported outcomes

$\mathrm{T}$ HE enactment of the Patient Protection and Affordable Care Act in 2010 established the foundation for the national standardization of health care delivery, ultimately leading to wide-ranging legislative oversight and determination of health care value..$^{18}$ Given the rapid growth and associated cost of surgical procedures, there is increasing interest in producing objective data to determine the quality of care and inform the allocation of health care resources.

Patient-reported outcomes (PROs) are gaining a central role in evaluating the effectiveness of surgical interventions and changes in disease trajectory. More importantly, PROs are more frequently incorporated in clinical trials as the primary outcome comparing health interventions for chronic diseases. ${ }^{26,27}$ However, a challenge to interpreting the meaning of improvement in PROs is that the extent of change in a numerical score lacks a direct meaning or clinical significance. ${ }^{14}$ The concept of minimum clinically important difference (MCID) has been put forth as the smallest improvement in the PRO needed to achieve a level of clinical improvement. In other words, health-related quality-of-life and pain scores above MCID potentially represent important changes from a patient's perspective.

PROs are particularly important in the realm of spinal surgery. With the advent of individualized medicine, there is increasing need to provide state-of-the-art surgical spine interventions tailored to the particular needs and symptoms of the patient population. Defining the MCID for specific spinal pathologies represents an important step in that direction. Previously published studies have attempted to define MCID thresholds for spinal stenosis, ${ }^{25}$ pseudarthrosis, ${ }^{22}$ adjacent-segment disease, ${ }^{16}$ and spinal cord stimulation for failed back surgery syndrome. ${ }^{24}$ However, literature on the MCID for patients with degenerative lumbar spondylolisthesis is scarce, and most of the published studies are limited by their single-institution nature and small sample size. In light of this knowledge gap, we sought to determine the MCID values of functional outcomes in patients who underwent surgery for degenerative spondylolisthesis.

\section{Methods}

\section{Data Source}

We queried the Quality Outcomes Database (QOD) registry for the period from July 2014 through December $2015 .^{20}$ The QOD is a prospective registry that is designed to capture 12-month outcomes with the aim of improving efficiency and quality of care for the most commonly performed spinal surgical procedures. ${ }^{17}$ Preoperative radiographs, standing or dynamic, were evaluated by surgeons at each participating site to confirm the diagnosis of spondylolisthesis. ${ }^{20}$ Patients who underwent elective surgery for Meyerding classification ${ }^{19}$ grade I spondylo- listhesis through a posterior-only approach were included in the analysis.

\section{Patient-Reported Outcomes}

The primary outcome measures were assessed at 12 months using validated questionnaires-the Oswestry Disability Index (ODI $)^{8}$ and the EQ-5D ${ }^{7}$ (EuroQoL Group) preference-based measure of health status-as well as a numeric rating scale (NRS) for back and leg pain (NRSBP and NRS-LP, respectively). As previously described, ${ }^{4}$ the ODI change scores and the pain scale scores were multiplied by -1 , so that a positive change would reflect an improvement in all the PRO scores.

\section{Anchor-Based Approaches}

Anchor-based methods use an external indicator, i.e., an "anchor," to assign patients into various groups that reflect different changes in their clinical or health status (large positive vs small positive change vs no change). The anchor can be either a clinical variable, such as a laboratory marker, physiological measure, or clinician rating, or a patient-reported variable, such as a score on the SF-36, which is a generic health-related quality-of-life instrument. ${ }^{26}$

In this study, we used the North American Spine Society (NASS) satisfaction scale as the anchor. ${ }^{6}$ The NASS satisfaction battery is a 4-point scale with the following responses: 1) "Surgery met my expectations," 2) "I did not improve as much as I had hoped but I would undergo the same operation for the same results," 3) "Surgery helped but I would not undergo the same operation for the same results," and 4) "I am the same as or worse than before surgery."

Based on previously published descriptions, we designated patients in categories 1 and 2 as "satisfied" and those in categories 3 and 4 as "not satisfied" with their surgical treatment. ${ }^{20}$

\section{Distribution-Based Approaches}

Distribution-based methods are based on the theory that the MCID can be calculated based on the distribution of observed scores in a given sample. ${ }^{26}$ The following 4 methods were used in our analysis.

Half a Standard Deviation. The standard deviation (SD) describes the variability in a sample of patient scores. Norman and colleagues ${ }^{21}$ have reported that a value of 0.5 SD corresponds to the MCID across multiple different studies and outcomes. They demonstrated that $0.5 \mathrm{SD}$ represents the limit of the human mental discriminative capacity and is equal to 1 standard error of measurement (SEM) with a reliability of 0.75 .

Cohen's Effect Size. The effect size is a standardized measure of change that is defined as the difference in 
score from baseline to posttreatment divided by the SD of the baseline score. According to Cohen, ${ }^{3}$ an effect size of 0.2 is considered small, 0.5 moderate, and 0.8 large. To obtain the MCID, one multiplies the SD of the baseline score by $0.2 .^{5}$

Standard Error of Measurement. The standard error of measurement (SEM) is the variation in the scores due to the unreliability of the scale or measure used. Accordingly, a change in the PRO score smaller than the identified SEM is more likely to be the result of measurement error than a true observed change. ${ }^{26}$ The $\mathrm{SEM}$ is calculated as $\mathrm{SD} \times \sqrt{ }(1$ $-r$ ), where $r$ is the test-retest reliability coefficient. For the purposes of this study, we used the previously published reliability values of 0.90 for ODI, ${ }^{11} 0.90$ for EQ-5D, ${ }^{15}$ and 0.95 for the leg and back pain scales. ${ }^{15}$

Minimum Detectable Change. The minimum detectable change (MDC) is the smallest change that is considered above the measurement error. It is calculated as SEM $\times 1.96 \times \sqrt{2}$ for the $95 \%$ confidence interval. ${ }^{1,29}$ As previously published, the MCID should be at least as large as the calculated MDC. ${ }^{5}$

\section{Statistical Analysis}

Descriptive statistics (means with SDs for continuous variables and frequencies with proportions for categorical variables) were used to present demographic information and PRO scores. Between satisfied and not-satisfied patients, continuous variables were compared using 2-tailed, unpaired Student t-tests, while categorical variables were compared using Pearson's chi-square and Fisher's exact tests.

A receiver operating characteristic (ROC) curve was constructed for each PRO, plotting sensitivity against 1-specificity. Sensitivity is defined as the proportion of patients who report improvement based on the anchor and who have a PRO score above the MCID threshold value. Conversely, specificity is defined as the proportion of patients who do not report improvement based on the anchor and who have a PRO score below the MCID threshold value. $5,12,28$ The threshold value, i.e., change score between baseline and 12 months, that maximizes both sensitivity and specificity (Youden's index) was used to determine the ROC-based MCID. ${ }^{30}$ The analysis was repeated separately for the fusion and the laminectomy subgroups to investigate discrepancies in MCID values based on procedure type. Pearson's and Spearman's correlation coefficients were calculated to determine the association between baseline and change scores as well as the satisfaction anchor. As previously described, we used a correlation threshold of 0.30 to define an acceptable association between an anchor and a PRO change score. ${ }^{3,27}$ Analysis was conducted using $\mathrm{R}$ open-source software (version 3.3.2) and JMP Pro (version 12.2.0).

\section{Results}

A total of 441 patients from 11 participating sites were included in the analysis. The patients' mean age was 62 years (SD 11.2), and 58\% were female. Arthrodesis was performed in 361 cases, and 80 patients had laminectomy alone.

\section{Summary of PRO Scores}

Baseline and 1-year functional outcome scores are summarized in Table 1. Table 2 presents the change scores stratified by patient satisfaction status. The changes in functional outcome scores between baseline and 1 year were $23.5 \pm 17.4$ points for ODI, $0.24 \pm 0.23$ for EQ-5D, $4.1 \pm 3.5$ for NRS-LP, and $3.7 \pm 3.2$ for NRS-BP. The improvement in PRO scores was significantly higher $(\mathrm{p}<$ 0.001 for all PROs) for the satisfied group than for the not satisfied group.

\section{Determining the MCID}

Each calculation method yielded a different threshold value (Table 3). The average change calculation for the satisfied and not-satisfied groups consistently yielded the largest value and the effect size method yielded the smallest value. These ranged from 3.3 to 26.6 points for ODI, 0.04 to 0.3 for EQ-5D, 0.6 to 4.6 for NRS-LP, and 0.5 to 4.2 for NRS-BP, translating to a difference ranging from 7- to 8-fold depending on the PRO. Regarding ROC-based MCID, the area under the curve (AUC) for each PRO was as follows: ODI, 0.80; EQ-5D, 0.71; NRS-LP, 0.71, and NRS-BP, 0.75 . The average changes in ODI, NRS-LP, and NRS-BP were greater than the MCID as determined by the MDC (Fig. 1).

\section{Correlation With Anchor and Baseline Scores}

The association between the change scores and the responses to the anchor were all significant ( $p<0.001$ for all), with greater correlation with respect to ODI $(r=0.45)$, followed by NRS-BP $(r=0.39)$, NRS-LP $(r=0.31)$, and EQ-5D $(r=0.30)$. In addition, large and significant correlations were found between the change and the baseline scores: -0.47 for ODI, 0.66 for EQ-5D, 0.55 for NRS-LP, and 0.56 for NRS-BP ( $<<0.001$ for all) (Table 4).

\section{Subgroup Analysis}

Forty-four patients (81\%) in the laminectomy group and 249 patients $(84 \%)$ in the fusion group answered that they were satisfied with the results of their surgical treatment. The results of the MCID calculation methods for the 2 groups are presented in Tables 5 and 6 . The ranges of MCID values for each PRO for the laminectomy patients were as follows: 3.3-21.4 points for ODI, 0.04-0.3 for EQ5D, $0.6-5.5$ for NRS-LP, and 0.6-3.9 for NRS-BP. For the fusion patients, the ranges were 3.2-27.5 points for ODI, 0.04-0.3 for EQ-5D, 0.5-4.5 for NRS-LP, and 0.5-4.2 for NRS-BP. Based on the MDC method, the MCID thresholds of the laminectomy subset (14.3 points for ODI, 0.2 points for EQ-5D, 1.7 points for NRS-LP, and 1.9 points for NRS-BP) were comparable to those of the fusion subset (14 points for ODI, 0.2 points for EQ-5D, 1.7 points for NRS-LP, and 1.5 points for NRS-BP) as well as those of the entire series.

\section{Discussion}

In the present study we analyzed data from 441 cases involving patients enrolled in a multi-institutional spine registry who underwent posterior lumbar surgery for grade I degenerative lumbar spondylolisthesis. Adhering 
TABLE 1. Baseline and 1-year PRO scores

\begin{tabular}{|c|c|c|c|c|}
\hline Outcome Measure & No. of Pts w/ Available Data & All Ops $(n=441)$ & Laminectomy Alone $(n=80)$ & Fusion $(n=361)$ \\
\hline \multicolumn{5}{|l|}{ ODI } \\
\hline Baseline & 441 & $44.1(16.3)$ & $36.8(16.3)$ & $45.8(15.8)$ \\
\hline 1-yr postop & 356 & $21.0(17.5)$ & $17.8(13.5)$ & $21.6(18.1)$ \\
\hline \multicolumn{5}{|l|}{$E Q-5 D$} \\
\hline Baseline & 441 & $0.54(0.22)$ & $0.56(0.22)$ & $0.53(0.22)$ \\
\hline 1-yr postop & 342 & $0.78(0.18)$ & $0.80(0.16)$ & $0.78(0.19)$ \\
\hline \multicolumn{5}{|l|}{ NRS-LP } \\
\hline Baseline & 441 & $6.65(2.74)$ & $6.61(2.81)$ & $6.63(2.73)$ \\
\hline $1-y r$ postop & 354 & $2.49(3.04)$ & $2.21(2.58)$ & $2.54(3.12)$ \\
\hline \multicolumn{5}{|l|}{ NRS-BP } \\
\hline Baseline & 441 & $6.79(2.60)$ & $5.94(3.00)$ & $6.98(2.47)$ \\
\hline 1-yr postop & 355 & $3.06(2.76)$ & $2.57(2.52)$ & $3.16(2.79)$ \\
\hline NASS satisfaction & 352 & & & \\
\hline $1, \mathrm{n}(\%)$ & & $239(67.9)$ & $38(70.4)$ & $201(67.4)$ \\
\hline $2, \mathrm{n}(\%)$ & & $54(15.3)$ & $6(11.1)$ & $48(16.1)$ \\
\hline $3, \mathrm{n}(\%)$ & & $35(9.94)$ & $7(13.0)$ & $28(9.40)$ \\
\hline $4, \mathrm{n}(\%)$ & & $24(6.82)$ & $3(5.56)$ & $21(7.05)$ \\
\hline
\end{tabular}

Pts $=$ patients.

Data are presented as mean (SD) unless otherwise indicated.

to the methods described in the MCID literature and suggested by expert panels in evidence-based medicine, ${ }^{10,13,26}$ we found significant variations (7- to 8-fold) in the MCID values for ODI, NRS-LP, and NRS-BP. These ranged from 3.3 to 26.6 points for ODI, 0.04 to 0.3 for EQ-5D, 0.6 to 4.6 for NRS-LP, and 0.5 to 4.2 for NRS-BP. The smallest value was derived from the effect size calculation, whereas the greatest value was the average change within satisfied patients.

To date, no consensus has been reached as to which MCID calculation method is superior. As Copay and colleagues have highlighted, a sound MCID value should 1) be at least larger than the measurement error and 2) correspond to the patient perception of importance of the change. ${ }^{4}$ Based on these attributes, MDC seems to be the superior calculation method for determining an MCID threshold. First, the choice of MDC as MCID has the advantage of excluding potential MCID thresholds that are within the measurement error. Second, it was closest to the average change difference between the satisfied and not-

TABLE 2. Change in outcome scores by satisfaction scale answers

\begin{tabular}{lrcc}
\hline $\begin{array}{l}\text { Outcome } \\
\text { Measure }\end{array}$ & All $(n=352)$ & $\begin{array}{c}\text { Not Satisfied } \\
(n=59)\end{array}$ & $\begin{array}{c}\text { Satisfied } \\
(n=293)\end{array}$ \\
\hline ODI & $23.53(17.4)$ & $8.71(14.6)$ & $26.53(16.4)$ \\
\hline EQ-5D & $0.24(0.23)$ & $0.10(0.22)$ & $0.26(0.22)$ \\
\hline NRS-LP & $4.14(3.45)$ & $2.00(3.15)$ & $4.56(3.35)$ \\
\hline NRS-BP & $3.70(3.18)$ & $1.45(2.19)$ & $4.14(3.15)$ \\
\hline
\end{tabular}

Data are presented as mean (SD) change in score for the specified outcome measure. The differences between groups were highly statistically significant $(p<0.001)$ for all comparisons. satisfied patients. Based on this method, the MCID values are 14.3 points for ODI, 0.2 points for EQ-5D, 1.7 points for NRS-LP, and 1.6 points for NRS-BP for patients who underwent posterior lumbar surgery for grade I degenerative lumbar spondylolisthesis. The percentage of patients who achieved MCID at 1 year was $71 \%$ for ODI, $58 \%$ for EQ-5D, 79\% for NRS-LP, and 76\% for NRS-BP.

Controversy also exists regarding the optimal anchor to choose. In this study, we used satisfaction with surgical treatment as an external indicator to calculate the MCID. Copay and colleagues found similar values when they used the health transition item (HTI) of the SF-36 compared with the Satisfaction with Results scale, which was constructed as a second anchor from patients' responses to 5 questions. ${ }^{4}$ In another study, the authors found the AUC to be slightly higher for the HTI anchor (0.73 vs 0.69 ), thus concluding that HTI is a more accurate anchor than satisfaction. ${ }^{23}$ Interestingly, MDC showed the least discrepancy between the 2 anchors. However, a potential drawback

TABLE 3. MCID determinations

\begin{tabular}{lcccc}
\hline \multicolumn{1}{c}{ Method } & ODI & EQ-5D & NRS-LP & NRS-BP \\
\hline Anchor based & & & & \\
\hline$\quad$ Average change & 26.6 & 0.3 & 4.6 & 4.2 \\
\hline Change difference & 17.4 & 0.2 & 2.6 & 2.7 \\
\hline$\quad$ ROC curve derived & 21.5 & 0.1 & 4.5 & 3.5 \\
\hline Distribution based & & & & \\
\hline Half SD & 8.1 & 0.11 & 1.4 & 1.3 \\
\hline Small Cohen's effect size $(0.2)$ & 3.3 & 0.04 & 0.6 & 0.5 \\
\hline 1 SEM & 5.1 & 0.1 & 0.6 & 0.6 \\
\hline MDC $(95 \% \mathrm{Cl})$ & 14.3 & 0.2 & 1.7 & 1.6 \\
\hline
\end{tabular}



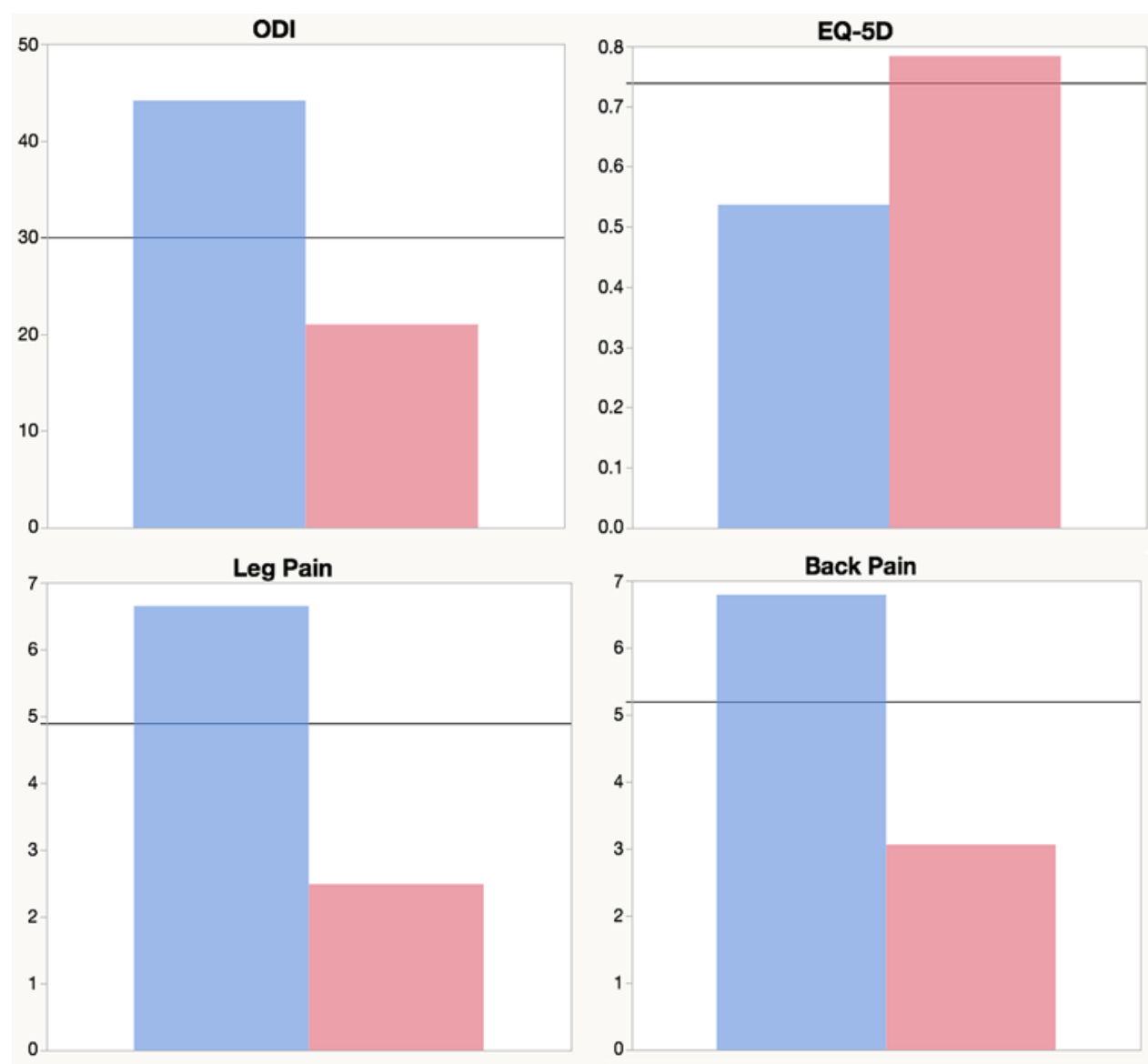

FIG. 1. Graphs showing the mean PRO scores at baseline (blue bars) and at 12 months' follow-up (pink bars) for the entire cohort. The black line represents the value of the MCID as determined by the MDC subtracted from the preoperative average score.

of a general health assessment questionnaire is that patient assessment may be unrelated to the condition investigated in the study. For example, patients may report lack of improvement in their health compared with their health status 1 year previously, but this may actually be due to a health issue that is not related to their back surgery.

Our study is the largest to date to investigate the MCID for patients surgically treated for spondylolisthesis. Parker and colleagues ${ }^{23}$ reported on 45 patients who underwent transforaminal interbody fusion (TLIF) for lumbar spondylolisthesis in a single, tertiary-referral academic center. Based on the MDC, they found the MCID values to be 14.9 points for ODI, 0.46 for EQ-5D, 2.8 points for the leg pain visual analog scale (VAS), and 2.1 points for the back pain VAS. In contrast, in an analysis by Copay and colleagues of 454 patients enrolled in the Lumbar Spine

TABLE 4. Correlation coefficients of score changes with baseline scores

\begin{tabular}{cccc}
\hline Outcome Measure & Baseline PRO & Satisfaction Anchor & $p$ Value \\
\hline ODI & 0.47 & 0.45 & $<0.001$ \\
\hline EQ-5D & 0.70 & 0.30 & $<0.001$ \\
\hline NRS-LP & 0.55 & 0.31 & $<0.001$ \\
\hline NRS-BP & 0.56 & 0.39 & $<0.001$ \\
\hline
\end{tabular}

Study Group who underwent lumbar spine surgery for a multitude of spine conditions, the calculated MCID values were 12.8 points for ODI, 1.2 points for back pain, and 1.6 points for leg pain. ${ }^{4}$ Our proposed thresholds are closer to those seen in the latter study, probably due to the large sample size, which reduced the noise level and allowed for more conservative estimates.

Since MCID values are also procedure specific, we conducted subgroup analyses for patients who underwent fusion and those who underwent laminectomy alone, with the hypothesis that greater change is needed based on in-

TABLE 5. MCID determinations for laminectomy-alone group

\begin{tabular}{lrrrr}
\hline \multicolumn{1}{c}{ Method } & ODI & EQ-5D & NRS-LP & NRS-BP \\
\hline Anchor based & & & & \\
\hline$\quad$ Average change & 21.4 & 0.3 & 4.8 & 3.8 \\
\hline Change difference & 12.6 & 0.1 & 2.4 & 3.9 \\
\hline$\quad$ ROC curve derived & 9.0 & 0.1 & 5.5 & 2.5 \\
\hline Distribution based & & & & \\
\hline$\quad$ Half SD & 8.2 & 0.1 & 1.4 & 1.5 \\
\hline Small Cohen's effect size $(0.2)$ & 3.3 & 0.04 & 0.6 & 0.6 \\
\hline 1 SEM & 5.2 & 0.07 & 0.6 & 0.7 \\
\hline MDC $(95 \% \mathrm{Cl})$ & 14.3 & 0.2 & 1.7 & 1.9 \\
\hline
\end{tabular}


TABLE 6. MCID determinations for fusion group

\begin{tabular}{lcccc}
\hline \multicolumn{1}{c}{ Method } & ODI & EQ-5D & NRS-LP & NRS-BP \\
\hline Anchor based & & & & \\
\hline$\quad$ Average change & 27.5 & 0.3 & 4.5 & 4.2 \\
\hline Change difference & 18.3 & 0.2 & 2.6 & 2.5 \\
\hline$\quad$ ROC curve derived & 21.5 & 0.2 & 4.5 & 3.5 \\
\hline Distribution based & & & & \\
\hline$\quad$ Half SD & 7.9 & 0.1 & 1.4 & 1.2 \\
\hline Small Cohen's effect size $(0.2)$ & 3.2 & 0.04 & 0.5 & 0.5 \\
\hline 1 SEM & 5.0 & 0.07 & 0.6 & 0.6 \\
\hline MDC $(95 \% \mathrm{Cl})$ & 13.9 & 0.2 & 1.7 & 1.5 \\
\hline
\end{tabular}

creasing invasiveness. The MCID values in the laminectomy-alone group were similar to those in the fusion group as well as the entire cohort. Similarly, when Parker and colleagues $^{23}$ analyzed the subset of cases in which patients underwent minimally invasive TLIF $(n=15)$ separately, they found the MCID thresholds to be comparable to those of the entire series. Therefore, it appears that MCID values depend more on the surgical diagnosis than on the procedure type.

The findings of this study also have notable implications with regard to health policy and resource utilization. Given the increasing demand for surgical quality measures in health care, the percentage of patients reaching MCID can serve as an appropriate marker for screening interventions that might be inefficient and add unnecessary financial burden to the health care system. Consequently, this could lead to significant cost reductions and prudent resource utilization, which is particularly important in the current era of budget constraints. Substantial clinical benefit might also represent an appropriate target goal during preoperative counseling and could serve as a primary outcome for studies that aim to evaluate the comparative and cost effectiveness of 2 surgical procedures. ${ }^{2,9}$ However, it is important to note that MCID thresholds should not be the sole target for determining surgical success. Several other parameters, including clinical variables (e.g., return to work and postoperative utilization of analgesic medication) and radiographic measures (e.g., fusion rate and correction of foraminal height), may be needed to define surgical success.

\section{Limitations}

Our study has several limitations. First, only 1 anchor (i.e., patient satisfaction with surgical treatment) was available to be used in the present analysis. Therefore, we could not assess consistency of results across different anchors. Nevertheless, Copay and colleagues ${ }^{4}$ demonstrated that the correlation between the Satisfaction with Results scale and PRO change scores was higher compared with the HTI anchor of the SF-36. Thus, it is unlikely that a different anchor would have a significant impact on our findings. Second, we calculated MCID for 12 months of follow-up and therefore cannot provide insight into longer-term functional outcome scores. It is possible that MCID values might vary based on the follow-up time frame. Third, an inherent limitation of MCID is that it likely varies based on starting point. This in particular seems to matter for ODI scores, as patients with greater preoperative disability require greater change to achieve satisfaction, whereas leg and back pain seem to be less sensitive to this effect. Fourth, our study is based on prospectively collected data from a registry, and these data are primarily collected for quality improvement and reporting rather than research purposes. Finally, MCID values do not take into account cost data. Given the rising pressure from policy makers and thirdparty payors to curb national health expenditures, cost of treatment needs to be incorporated into future analyses as well. ${ }^{24}$ Recently, the concept of minimal cost-effective difference has been suggested as the smallest improvement in an outcome instrument that is associated with a costeffective response to surgery. ${ }^{24}$

\section{Conclusions}

In this study, we analyzed PRO data from 441 patients enrolled in a national, prospective registry who underwent posterior surgery for grade I degenerative lumbar spondylolisthesis. The MDC approach appeared to be most appropriate for calculating MCID, because it provided a threshold above the measurement error and it was closest to the mean change difference between satisfied and notsatisfied patients. Based on the MDC method, the MCID values are 14.3 points for ODI, 0.2 points for EQ-5D, 1.7 points for NRS-LP, and 1.6 points for NRS-BP.

\section{Acknowledgments}

We would like to thank all of the site research coordinators for their help with data extraction and validation. We would also like to thank the Neurosurgery Research and Education Foundation for its financial support of this work.

\section{References}

1. Beaton DE, Bombardier C, Katz JN, Wright JG, Wells G, Boers M, et al: Looking for important change/differences in studies of responsiveness. J Rheumatol 28:400-405, 2001

2. Carreon LY, Glassman SD, Campbell MJ, Anderson PA: Neck Disability Index, Short Form-36 Physical Component Summary, and pain scales for neck and arm pain: the minimum clinically important difference and substantial clinical benefit after cervical spine fusion. Spine J 10:469-474, 2010

3. Cohen J: Statistical Power Analysis for the Behavioral Sciences. Hillsdale, NJ: Lawrence Earlbaum Associates, 1988

4. Copay AG, Glassman SD, Subach BR, Berven S, Schuler TC, Carreon LY: Minimum clinically important difference in lumbar spine surgery patients: a choice of methods using the Oswestry Disability Index, Medical Outcomes Study questionnaire Short Form 36, and pain scales. Spine J 8:968-974, 2008

5. Copay AG, Subach BR, Glassman SD, Polly DW Jr, Schuler TC: Understanding the minimum clinically important difference: a review of concepts and methods. Spine J 7:541-546, 2007

6. Daltroy LH, Cats-Baril WL, Katz JN, Fossel AH, Liang MH: The North American Spine Society Lumbar Spine Outcome Assessment Instrument: reliability and validity tests. Spine (Phila Pa 1976) 21:741-749, 1996

7. EuroQol Group: EuroQol-a new facility for the measurement of health-related quality of life. Health Policy 16:199208, 1990

8. Fairbank JC, Pynsent PB: The Oswestry Disability Index. Spine (Phila Pa 1976) 25:2940-2952, 2000 
9. Glassman SD, Copay AG, Berven SH, Polly DW, Subach BR, Carreon LY: Defining substantial clinical benefit following lumbar spine arthrodesis. J Bone Joint Surg Am 90:18391847,2008

10. Guyatt GH, Osoba D, Wu AW, Wyrwich KW, Norman GR: Methods to explain the clinical significance of health status measures. Mayo Clin Proc 77:371-383, 2002

11. Hägg O, Fritzell P, Nordwall A: The clinical importance of changes in outcome scores after treatment for chronic low back pain. Eur Spine J 12:12-20, 2003

12. Jaeschke R, Guyatt GH, Sackett DL: Users' guides to the medical literature. III. How to use an article about a diagnostic test. B. What are the results and will they help me in caring for my patients? JAMA 271:703-707, 1994

13. Jaeschke R, Singer J, Guyatt GH: Measurement of health status. Ascertaining the minimal clinically important difference. Control Clin Trials 10:407-415, 1989

14. Kulkarni AV: Distribution-based and anchor-based approaches provided different interpretability estimates for the Hydrocephalus Outcome Questionnaire. J Clin Epidemiol 59:176-184, 2006

15. McDowell I: Measuring Health: A Guide to Rating Scales and Questionnaires. New York: Oxford University Press, 2006

16. McGirt M, Parker S, Adogwa O, Mendenhall S, Shau D, Cheng J, et al: Determination of minimum clinically important difference (MCID) in pain, disability, and quality of life after extension of fusion for adjacent segment disease. Spine J 10 Suppl:S50, 2011 (Abstract)

17. McGirt MJ, Speroff T, Dittus RS, Harrell FE Jr, Asher AL: The National Neurosurgery Quality and Outcomes Database $\left(\mathrm{N}^{2} \mathrm{QOD}\right)$ : general overview and pilot-year project description. Neurosurg Focus 34(1):E6, 2013

18. Menger RP, Guthikonda B, Storey CM, Nanda A, McGirt M, Asher A: Neurosurgery value and quality in the context of the Affordable Care Act: a policy perspective. Neurosurg Focus 39(6):E5, 2015

19. Meyerding HW: Diagnosis and roentgenologic evidence in spondylolisthesis. Radiology 20:108-120, 1933

20. Mummaneni PV, Bisson EF, Kerezoudis P, Glassman S, Foley $\mathrm{K}$, Slotkin JR, et al: Minimally invasive versus open fusion for Grade I degenerative lumbar spondylolisthesis: analysis of the Quality Outcomes Database. Neurosurg Focus 43(2): E11, 2017

21. Norman GR, Sloan JA, Wyrwich KW: Interpretation of changes in health-related quality of life: the remarkable universality of half a standard deviation. Med Care 41:582-592, 2003

22. Parker SL, Adogwa O, Mendenhall SK, Shau DN, Anderson WN, Cheng JS, et al: Determination of minimum clinically important difference (MCID) in pain, disability, and quality of life after revision fusion for symptomatic pseudoarthrosis. Spine J 12:1122-1128, 2012

23. Parker SL, Adogwa O, Paul AR, Anderson WN, Aaronson $\mathrm{O}$, Cheng JS, et al: Utility of minimum clinically important difference in assessing pain, disability, and health state after transforaminal lumbar interbody fusion for degenerative lumbar spondylolisthesis. J Neurosurg Spine 14:598-604, 2011

24. Parker SL, McGirt MJ: Determination of the minimum improvement in pain, disability, and health state associated with cost-effectiveness: introduction of the concept of minimum cost-effective difference. Neurosurgery 76 (Suppl 1):S64S70, 2015

25. Parker SL, Mendenhall SK, Shau DN, Adogwa O, Anderson WN, Devin CJ, et al: Minimum clinically important difference in pain, disability, and quality of life after neural decompression and fusion for same-level recurrent lumbar stenosis: understanding clinical versus statistical significance. J Neurosurg Spine 16:471-478, 2012

26. Revicki D, Hays RD, Cella D, Sloan J: Recommended methods for determining responsiveness and minimally important differences for patient-reported outcomes. J Clin Epidemiol 61:102-109, 2008

27. Sloan JA, Dueck A: Issues for statisticians in conducting analyses and translating results for quality of life end points in clinical trials. J Biopharm Stat 14:73-96, 2004

28. van der Roer N, Ostelo RWJG, Bekkering GE, van Tulder MW, de Vet HCW: Minimal clinically important change for pain intensity, functional status, and general health status in patients with nonspecific low back pain. Spine (Phila Pa 1976) 31:578-582, 2006

29. Wells G, Beaton D, Shea B, Boers M, Simon L, Strand V, et al: Minimal clinically important differences: review of methods. J Rheumatol 28:406-412, 2001

30. Youden WJ: Index for rating diagnostic tests. Cancer 3:3235,1950

\section{Disclosures}

Bisson: consultant for nView. Glassman: employee of Norton Healthcare; patent holder with, consultant for, and royalties from Medtronic; NuVasive (provides funds directly to the database company; no funds are paid directly to individual or institution); and past president of the Scoliosis Research Society. Foley: consultant for Medtronic; direct stock ownership in Discgenics, Medtronic, NuVasive, Spine Wave, and TrueVision; patent holder with Medtronic and NuVasive; Board of Directors, Medtronic, Discgenics, and TrueVision. Potts: consultant for and royalties from Medtronic. Slotkin: consultant for Stryker Spine and Medtronic. C Shaffrey: direct stock ownership in NuVasive; consultant for Medtronic, NuVasive, and Zimmer Biomet; patent holder with Medtronic, NuVasive, and Zimmer Biomet. Coric: consultant for Spine Wave, Stryker, Medtronic, Globus Medical, and Premia Spine; and direct stock ownership in Spine Wave, Premia Spine, and Spinal Kinetics. Knightly: NPA Board of Directors. Park: royalties from Globus; consultant for Globus, NuVasive, Zimmer Biomet, and Medtronic. Fu: consultant for SIBONE. Devin: consultant for Stryker Spine and Medtronic; clinical or research support for study described, Stryker Spine. Mummaneni: consultant for DePuy Spine, Globus, and Stryker; direct stock ownership in Spinicity/ISD and Globus; clinical/research support for this study from NREF; royalties from DePuy Spine, Thieme Publishers, and Springer Publishers; grant from AOSpine; and honoraria from Globus.

\section{Author Contributions}

Conception and design: Bydon, Asher, Kerezoudis, Mummaneni, Bisson, Glassman, Foley, Slotkin, Potts, M Shaffrey, C Shaffrey, Coric, Knightly, Park, Fu, Devin, Chotai, Chan. Acquisition of data: Bydon, Asher, Kerezoudis, Mummaneni, Bisson, Glassman, Foley, Slotkin, Potts, M Shaffrey, C Shaffrey, Coric, Knightly, Park, Fu, Chotai, Chan, Virk. Analysis and interpretation of data: Asher. Drafting the article: Bydon, Kerezoudis, Devin, Archer, Chotai, Virk. Critically revising the article: all authors. Reviewed submitted version of manuscript: Asher, Kerezoudis, Mummaneni, Bisson, Glassman, Foley, Slotkin, Potts, M Shaffrey, C Shaffrey, Coric, Knightly, Park, Fu, Devin, Archer, Chotai, Chan, Virk. Statistical analysis: Kerezoudis. Study supervision: Bydon, Asher, Mummaneni, Bisson.

\section{Correspondence}

Mohamad Bydon: Mayo Clinic, Rochester, MN. bydon. mohamad@mayo.edu. 